\title{
Fokus auf die Rahmenbedingungen
}

\author{
Krankenhaus-Infektionen wirksam vorbeugen
}

Etwa ein Viertel der Todesfälle durch nosokomiale, also im Krankenhaus erworbene, Infektionen könnte durch intensive Hygiene- und Kontroll-Programme verhindert werden, stellt das European Centre for Disease Prevention and Control (ECDC) fest. Dazu bedarf es sowohl mehr Aufmerksamkeit von Seiten des Gesundheitspersonals aber auch der Führungskräfte und Entscheidungsträger im Krankenaus und in den politischen Positionen. Eine Expertenrunde der Initiative „Sicherheit im OP“ und der „Plattform Patientensicherheit" diskutierte Anfang des vergangenen Sommers in Wien über Rahmenbedingungen der Prävention.

Mindestens 37.000 Menschen sterben laut ECDC europaweit an nosokomialen Infektionen, die für zusätzlich 110.000 Todesfälle zumindest mitverantwortlich sind. Für Österreich bedeutet das jährlich etwa 2.400 solcher Todesfälle. „ECDC-Auswertungen der Punktprävalenzstudie 2012 zeigen, dass wir in Österreich insgesamt im europäischen Durchschnitt liegen", berichtete Univ.-Prof. Dr. Elisabeth Presterl, Universitätsklinik für Krankenhaushygiene und Infektionskontrolle, Wien.

Zwei Beispiele aus Dänemark und Deutschland zeigten erfolgreiche Initiativen zur Reduktion nosokomialer Infektionen. Beide Beispiele verdeutlichen die Notwendigkeit, dass der politische Wille für entsprechende Präventionsmaßnahmen gegeben sein muss, um den Mitarbeitern in der Praxis den entsprechenden Rückhalt zu geben. Im Zusammenwirken zwischen „Top-Down“ und „Bottom Up“ wurden auf der organisatorischen Grundlage einer „Task Force“ nach drei Jahren eine mehr als 50-prozentige Reduktion bei Lungenentzündungen durch Beatmungsgeräte in allen Intensivstationen erzielt werden, berichtete Dr. Bettina Lundgren, Rigshospitalet, Kopenhagen: „Einige Krankenhäuser hatten 30 Prozent weniger Infektionen mit Clostridium difficile." Teil der Maßnahmen war die Gründung einer speziellen Datenbank mit dem Ziel, automatisch und kontinuierlich SurveillanceDaten über Krankenhaus-Infektionen zu generieren und Transparenz zu schaffen, damit sich nicht nur Mikrobiologen, Pfle- gepersonal und Ärzte informieren können, sondern auch Politiker und Bürger. Die Erfolge unterscheiden sich in einzelnen Bereichen ebenso wie zwischen den Krankenhäusern. So lautete auch eine wichtige Erkenntnis, dass Interventionen an den lokalen Kontext angepasst werden müssen.

\section{Hygiene ist Chefsache}

Der regionale Zusammenschluss von multiprofessionellen Akteuren im Gesundheitswesen zum MRSA-Netzwerks Osnabrück setzte sich als Ziel die Zahl von MRSA-Neuinfektionen zu reduzieren. Grundprämisse ist die Einstufung von Hygiene als politisch vorrangig und daher Chefsache. Konkret bedeutet das, so Dr. Gerhard Bojara, Leiter des Gesundheitsdienstes für Landkreis und Stadt Osnabrück und Projektleiter: „Leiter medizinischer Einrichtungen müssen das Hygienemanagement mittragen, und auch die erforderlichen Personal- und Finanzressourcen zur Verfügung zu stellen." Eine Erkenntnis im achten Jahr der Initiative: Netzwerke sind keine Selbstläufer, sondern müssen am Leben erhalten werden. Das bedeutet Arbeit - die sich letztlich lohnt. Zusätzlich zu den von der Netzwerk-Führung entwickelten Maßnahmen profitiert die Initiative auch vom Erfahrungsaustausch der Netzwerk-Partner.

\section{Fehlerquelle Arbeitsüberlastung}

Da internationale Untersuchungen einen direkten Zusammenhang zwischen einer hohen Arbeitsdichte des Gesundheitspersonals und dem Risiko einer Krankenhausinfektion zeigen, gelte es, so DGKP Josef Zellhofer, Vorsitzender der ÖGBFachgruppenvereinigung für Gesundheits- und Sozialberufe, ausreichend Personal bereitzustellen. In Österreich haben allerdings kontinuierlich steigende Anforderungen zu einer drastischen Verdichtung in der Arbeit geführt.

Mit dem Instrument von SurveillanceSystemen sollen auch nosokomiale Infektionen erhoben werden, um Häufigkeit, Entwicklungen und den Erfolg von Maßnahmen überprüfen zu können. „Alle Mitarbeiter sollten die Daten kennen, diese sollten mit dem Hygieneteam besprochen werden, Auffälligkeiten sollten überprüft und dann die richtigen Konsequenzen daraus gezogen werden“, erklärte Dr. Brigitte Ettl, Ärztliche Direktorin am KH Hietzing und Präsidentin der Österreichischen Plattform Patientensicherheit. Allerdings ist die Teilnahme an derartigen Plattformen in Österreich durchaus verbesserungsfähig, wie Univ.-Prof. Dr. Franz Allerberger, Bereichsleiter Humanmedizin in der AGES feststellte. Von den 96 allgemeinen Krankenhäusern und 48 Sanatorien nehmen derzeit 36 österreichische Intensivstationen am System Surveillance nosokomialer Infektionen auf Intensivstationen (ITS-KISS) teil. Und während das ECDC vorgibt, dass jede gesundheitsversorgende Einrichtung über ein Überwachungssystem für Erkrankungen durch C. difficile verfügen soll, waren hierzulande Ende 2015 für das Modul Surveillance System C. difficile assoziierte Diarrhoe (CDAD-KISS) nur zehn Einrichtungen registriert. Ähnlich ist die Situation bei den Modulen postoperative Wundinfektionen und Antibiotikaverbrauch.

Die Strategie des Gesundheitsministeriums (BMGF) bei der Infektionsprävention ist jedenfalls langfristig, so DDr. Reinhild Strauss, MSc, Leiterin der Abteilung Nosokomiale Infektionen und Antibiotikaresistenz im BMGF. Vor mehr als 20 Jahren begann man mit der intensiven Zusammenarbeit mit Experten in Krankenhäusern, Universitäten, Fachgesellschaften und anderen Gesundheitsbehörden, was unter anderem zur Leitlinien PROHYG (Organisation der Krankenhaushygiene) und dem österreichischen Resistenzbericht AURES führte.

Quelle: Presseaussendung „Sicherheit im OP“ und "Plattform Patientensicherheit“"

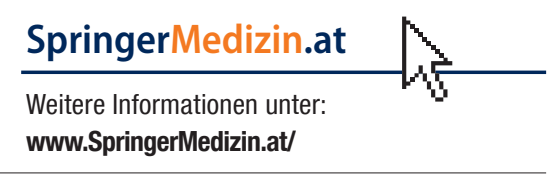

